\title{
Evolution of nuclear ground-state properties of neutron-deficient isotopes around $Z=82$ from precision mass measurements
}

\author{
Ch. Böhm,,${ }^{1, *}$ Ch. Borgmann,,${ }^{1 \dagger}$ G. Audi, ${ }^{2}$ D. Beck, ${ }^{3}$ K. Blaum, ${ }^{1}$ M. Breitenfeldt, ${ }^{4}$ R. B. Cakirli, ${ }^{1, \ddagger}$ T. E. Cocolios,${ }^{5,6}$ S. Eliseev, ${ }^{1}$ \\ S. George, ${ }^{7, \S}$ F. Herfurth, ${ }^{3}$ A. Herlert, ${ }^{6, \|}$ M. Kowalska, ${ }^{6}$ S. Kreim, ${ }^{1,6}$ D. Lunney, ${ }^{2}$ V. Manea, ${ }^{2}$ E. Minaya Ramirez, ${ }^{8,3, \S}$ \\ S. Naimi, ${ }^{2, \pi}$ D. Neidherr, ${ }^{1, * *}$ M. Rosenbusch, ${ }^{*}$ L. Schweikhard, ${ }^{9}$ J. Stanja, ${ }^{10, \dagger \dagger}$ M. Wang, ${ }^{2}$ R. N. Wolf, ${ }^{9,}$, and K. Zuber $^{10}$ \\ ${ }^{1}$ Max-Planck-Institut für Kernphysik, 69117 Heidelberg, Germany \\ ${ }^{2}$ CSNSM-IN2P3-CNRS, Université Paris-Sud, 91405 Orsay, France \\ ${ }^{3}$ GSI Helmholtzzentrum GmbH, 64291 Darmstadt, Germany \\ ${ }^{4}$ Instituut voor Kern- en Stralingsfysika, KU Leuven, 3001 Heverlee, Belgium \\ ${ }^{5}$ University of Manchester, School of Physics and Astronomy, Manchester M13 9PL, United Kingdom \\ ${ }^{6}$ CERN, Physics Department, 1211 Genève 23, Switzerland \\ ${ }^{7}$ NSCL, Michigan State University, East Lansing, Michigan 48824-1321, USA \\ ${ }^{8}$ Helmholtz-Institut Mainz, 55099 Mainz, Germany \\ ${ }^{9}$ Ernst-Moritz-Arndt Universität, Institut für Physik, 17487 Greifswald, Germany \\ ${ }^{10}$ Technische Universität Dresden, 01069 Dresden, Germany
}

(Received 26 June 2014; revised manuscript received 1 September 2014; published 7 October 2014)

\begin{abstract}
High-precision mass measurements of neutron-deficient $\mathrm{Tl}(A=184,186,190,193-195,198)$ isotopes as well as $\mathrm{Pb}(A=202,208), \operatorname{Fr}(A=207,208)$, and $\mathrm{Ra}(A=224)$ are performed with the Penning-trap mass spectrometer ISOLTRAP at ISOLDE/CERN. The improved precision of the mass data now allows the study of subtle odd-even effects. The gradual development of collectivity with the removal of protons from the magic $Z=82$ core is analyzed by combining the new mass results with nuclear charge-radii data and mean-field model predictions.
\end{abstract}

DOI: 10.1103/PhysRevC.90.044307

PACS number(s): 21.10.Dr, 07.75.+h, 21.60.-n

\section{INTRODUCTION}

The binding energy of a nucleus is one of the key observables for understanding its structure. Finite-difference mass formulas of the binding energy can be used to explore nuclear-structure effects. Illustrative is the commonly used two-neutron separation energy, $S_{2 n}$, which demonstrates the shell structure of nuclides by sharp drops after the filling of neutron shells. It is also sensitive to the onset of nuclear deformation, which changes the trends of $S_{2 n}$ and can lead, in particular cases, to an increase in $S_{2 n}$ with the addition of neutrons, as in the case of the neutron-rich $A \approx 100$ nuclides (see [1] and references therein). Combining the results of mass measurements with nuclear charge-radii information can provide even more detailed insights. In the case of neutron-

\footnotetext{
*christine.boehm@mpi-hd.mpg.de

${ }^{\dagger}$ Present address: Department of Physics and Astronomy, Uppsala University, Box 516, SE-751 05 Uppsala, Sweden.

${ }^{\ddagger}$ Present address: Department of Physics, University of Istanbul, 34134 Istanbul, Turkey.

${ }^{\S}$ Present address: Max-Planck-Institut für Kernphysik, 69117 Heidelberg, Germany.

"Present address: FAIR GmbH, 64291 Darmstadt, Germany.

"Present address: RIKEN Nishina Center for Accelerator-Based Science, 2-1 Hirosawa, Wako-shi, Saitama 351-0198, Japan.

** Present address: GSI Helmholtzzentrum GmbH, 64291 Darmstadt, Germany.

${ }^{\dagger \dagger}$ Present address: Max-Planck-Institut für Plasmaphysik, 85748 Garching, Germany.
}

deficient ${ }_{82} \mathrm{~Pb}$ isotopes the differential mean-square nuclear charge radii exhibit a regular odd-even staggering, meaning that the nuclear charge radii of odd $-N \mathrm{~Pb}$ isotopes are smaller than the average of the neighboring even- $N$ isotopes. With just a few protons less, however, the isotopic chains of mercury $\left({ }_{80} \mathrm{Hg}\right)$ and gold $\left({ }_{79} \mathrm{Au}\right)$ exhibit strong signs of deformation like shape staggering towards midshell around $N=104$ [2]. This unusual behavior of nuclear charge radii was interpreted as an indication of shape coexistence [3], apparently caused by intruder states arising from proton excitations across the $Z=82$ shell gap. Just over a decade ago, a most dramatic example of shape coexistence was found in the lead isotopes, namely, ${ }_{82}^{186} \mathrm{~Pb}$ at neutron number $N=104$ [4] with a spherical ground state and two deformed excited states within less than $700 \mathrm{keV}$ energy difference. Located between the spherical lead isotopes and the mercury isotopes, the thallium $\left({ }_{81} \mathrm{Tl}\right)$ isotopes are therefore particularly interesting.

The known nuclear charge radii and magnetic moments of the thallium isotopes point to a persistence of spherical configurations in the ground states down to $N=102[5,6]$. The $I=(9 / 2)$ even- $N$ thallium isomers have been interpreted as an intrusion of the proton $h_{9 / 2}$ orbital from above the $Z=82$ shell gap and their higher nuclear charge radius as a sign of increased deformation. The high accuracy of our data allows for study of the fine details of shape evolution in neutron-deficient $\mathrm{Tl}$ isotopes employing higher-order finitedifference mass formulas such as the so-called empirical pairing gap [7]. We compare our data to nuclear charge radii and microscopic calculations. Furthermore, the odd- $N$ $\left(7^{+}\right)$and the even- $N\left(9 / 2^{-}\right)$isomers of thallium are discussed. 
TABLE I. Information on the seven experimental campaigns at ISOLTRAP, including target and ion source combinations and investigated nuclides.

\begin{tabular}{lccccc}
\hline \hline Isotope(s) & Run & Date & $\begin{array}{c}\text { ISOLDE } \\
\text { ion energy }\end{array}$ & Target & Ion source \\
\hline${ }^{202} \mathrm{~Pb}$ & $\mathrm{~A}$ & May 2008 & $50 \mathrm{keV}$ & $\mathrm{UC}_{\mathrm{x}}$ & Hot plasma \\
${ }^{202,208 \mathrm{~Pb}}$ & $\mathrm{~B}$ & Oct. 2008 & $30 \mathrm{keV}$ & $\mathrm{UC}_{\mathrm{x}}$ & Plasma with quartz \\
${ }^{190,193 \mathrm{Tl}}$ & $\mathrm{C}$ & Apr. 2009 & $30 \mathrm{keV}$ & $\mathrm{UC}_{\mathrm{x}}$ & Hot plasma \\
${ }^{193-195} \mathrm{Tl}$ & $\mathrm{D}$ & Nov. 2010 & $40 \mathrm{keV}$ & $\mathrm{UC}_{\mathrm{x}}$ & Surface (W cavity) \\
${ }^{207,208} \mathrm{Fr},{ }^{224} \mathrm{Ra}$ & $\mathrm{E}$ & June 2011 & $50 \mathrm{keV}$ & $\mathrm{UC}_{\mathrm{x}}$ & Surface (Ta cavity) \\
${ }^{184,186,190} \mathrm{Tl},{ }^{208} \mathrm{Fr}$ & $\mathrm{F}$ & July 2011 & $50 \mathrm{keV}$ & $\mathrm{UC}_{\mathrm{x}}$ & Surface/RILIS (W cavity) \\
${ }^{198} \mathrm{Tl}$ & $\mathrm{G}$ & Oct. 2012 & $50 \mathrm{keV}$ & $\mathrm{UC}_{\mathrm{x}}$ & Surface (Ta cavity) \\
\hline \hline
\end{tabular}

In the following, the experimental setup is introduced (Sec. II) and the results of mass measurements (Sec. III) are discussed. The nuclear structure of the neutron-deficient $\mathrm{Tl}$ isotopes is studied (Sec. IV) by comparing thallium to its neighbors lead, mercury, and gold.

\section{EXPERIMENTAL SETUP}

The ISOLTRAP mass spectrometer [8,9], installed at the on-line isotope mass separator ISOLDE [10] at CERN, is designed for accurate, high-precision mass determination of exotic nuclides by cyclotron-frequency measurements in a Penning trap [11,12]. In the present study the ISOLTRAP spectrometer was used to investigate the nuclides ${ }_{184,186,190,193-195,198} \mathrm{Tl},{ }^{202,208} \mathrm{~Pb},{ }^{207,208} \mathrm{Fr}$, and ${ }^{224} \mathrm{Ra}$ produced at ISOLDE by impinging a $1.4-\mathrm{GeV}$ proton beam on a thick, high-temperature uranium carbide $\left(\mathrm{UC}_{\mathrm{x}}\right)$ target.

Employing either the high-resolution separator (HRS) or the general-purpose separator (GPS), the radionuclides are ionized, mass separated, and transported to different experiments at an energy of max. $60 \mathrm{keV}$. For technical reasons lower beam energies, in the range of 30-50 keV (see Table I), were chosen. One of the fixed setups is the ISOLTRAP experiment, which consists of four main parts as shown in Fig. 1. In the first trap, a linear radiofrequency quadrupole (RFQ) cooler and buncher [13], the quasicontinuous ion beam from ISOLDE is decelerated, cooled in a helium buffer-gas environment, and bunched. The ion bunches leave the first section with an energy of about $3.1 \mathrm{keV}$. Then the ions are guided through the multireflection time-of-flight mass separator (MR-TOF MS) [14], which was not employed in this work. After passing through the MR-TOF MS, the ions are transferred towards the tandem Penning-trap system.

Penning traps combine a strong homogeneous magnetic field and a weak electrostatic quadrupolar field to achieve confinement of charged particles. The mass determination of the stored ion is carried out by measuring the ion's cyclotron frequency,

$$
v_{\mathrm{c}}=\frac{1}{2 \pi} \cdot \frac{q B}{m_{\text {ion }}},
$$

where $q / m_{\text {ion }}$ is its charge-to-mass ratio and $B$ the magnetic field strength. To calibrate the magnetic field, the cyclotron frequency $v_{\mathrm{c}, \text { ref }}$ of an ion with a well-known mass is determined, before and after the measurement of the ion of interest.
The ${ }^{133} \mathrm{Cs}^{+}$reference ions used throughout this work were delivered from the off-line alkali ion source of ISOLTRAP (see Fig. 1).

The trajectory of an ion stored in a Penning trap is a superposition of three independent harmonic oscillator modes, characterized by the modified cyclotron frequency $v_{+}$, the axial frequency $v_{z}$, and the magnetron frequency $\nu_{-}$. At ISOLTRAP, the singly charged ions are first manipulated in the preparation Penning trap, which is a cylindrical trap for cooling and purification, employing the resonant buffer-gas cooling technique [15]: Ions of interest are centered and further transported, while unwanted species hit the aperture at the exit of the trap and are lost. Mass-resolving powers of the order of $R=m / \Delta m=10^{5}$ can be reached within a few hundred milliseconds. The isobarically purified ion bunches are extracted from the preparation trap and guided to the precision Penning trap, where the actual mass measurement takes place, employing the time-of-flight ion-cyclotron-resonance (TOF-ICR) technique [16]. In the precision trap first an azimuthal dipolar magnetron excitation is applied to bring the ions to a radius of about $0.7 \mathrm{~mm}$. Then the conversion to the cyclotron motion is achieved by applying a quadrupole excitation at $v_{\mathrm{c}}=v_{+}+v_{-}$. In the case of a full conversion, i.e., resonant excitation at the mass-dependent cyclotron frequency, the radial kinetic energy of the ion $E$ and, hence, its magnetic moment $\mu=E / B$ reach a maximum. The ions are extracted and transported to a microchannel plate (MCP 4 in Fig. 1) detector about $1.2 \mathrm{~m}$ downstream. The orbital magnetic moment of the ion couples to the magnetic field gradient and the ion is accelerated in the axial direction by the resulting force.

The TOF from the precision trap to the detector is recorded, and from a fit of the analytical resonance curve to the data, the frequency $v_{\mathrm{c}}$ is determined [17]. An example of a TOF-ICR of ${ }^{193} \mathrm{Tl}^{m}$ is shown in the inset in Fig. 1. The excitation time was $2 \mathrm{~s}$, yielding $R \approx 8.6 \times 10^{5}$. In addition to a cyclotron frequency measurement, the ions can be transferred to a decay-spectroscopy system to obtain additional information on isomers [18] (in the case of $\mathrm{Tl}$ see [19]).

\section{DATA ANALYSIS AND RESULTS}

The masses of the thallium, lead, francium, and radium isotopes presented in this paper have been determined in seven experimental campaigns. The details of the experimental 


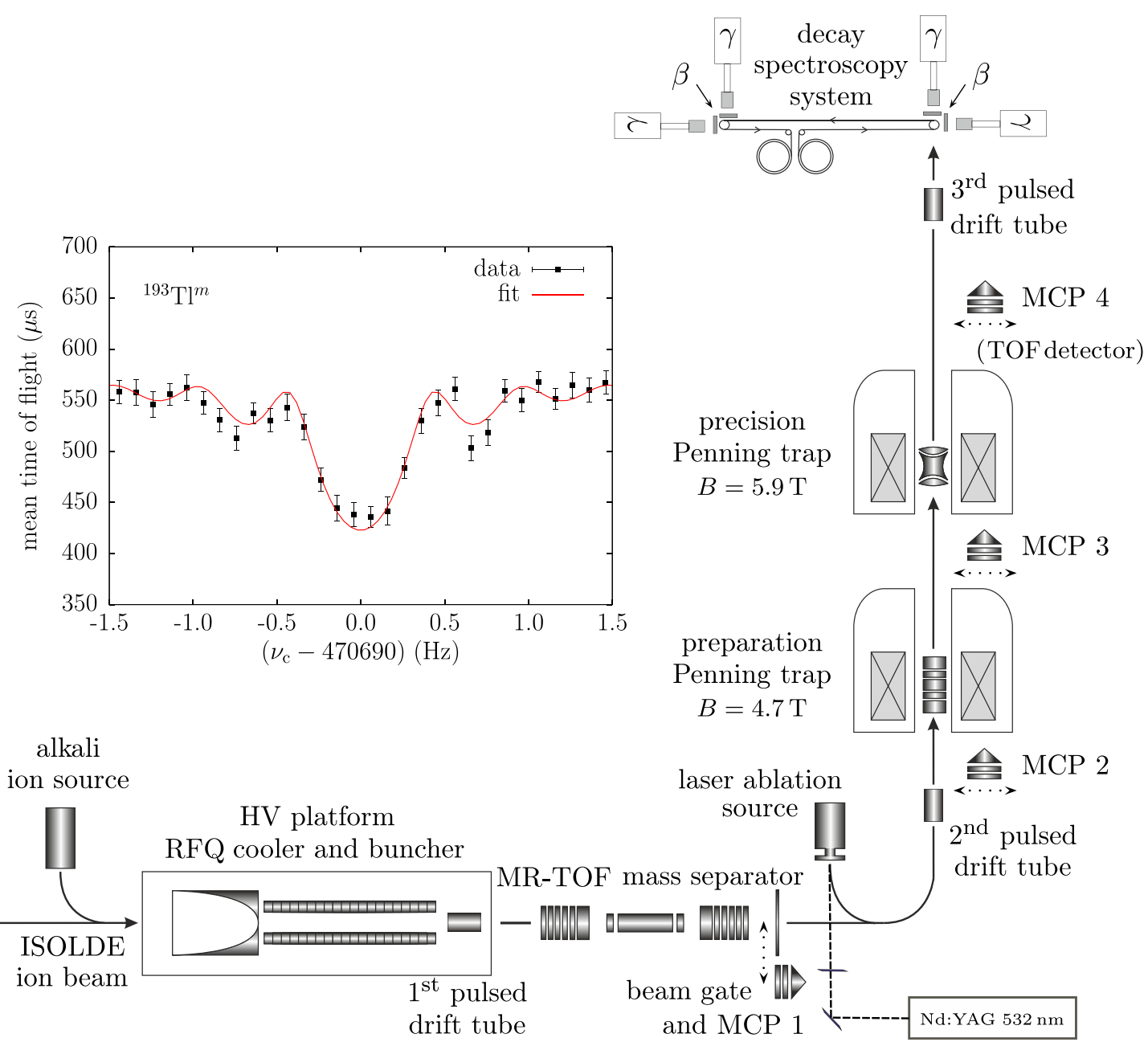

FIG. 1. (Color online) Schematic of the experimental setup: Ions coming from ISOLDE are accumulated in the RFQ cooler and buncher. Then the ion bunches pass the MR-TOF MS and are guided to the vertical part of the beamline into the preparation trap. After isobaric purification they are injected into the precision trap. Finally, the ions are transferred either to the MCP detector or to the decay-spectroscopy system. Inset: ${ }^{193} \mathrm{Tl}^{m}$ resonance taken with a $2 \mathrm{~s}$ excitation time.

campaigns including the date, beam energy, and target and ion-source combination are listed in Table I. To calibrate the magnetic field strength, reference measurements have been performed using ${ }^{133} \mathrm{Cs}^{+}$ions. The mass of the atom of interest $m$ is then calculated using the formula

$$
m=r \cdot\left(m_{\mathrm{ref}}-m_{\mathrm{e}}\right)+m_{\mathrm{e}}, \quad \text { with } \quad r=\frac{v_{\mathrm{c}, \mathrm{ref}}}{v_{\mathrm{c}}},
$$

where $m_{\mathrm{e}}$ is the electron mass [20] and $m_{\text {ref }}$ the atomic mass of the reference ion. For calculation of the mass values presented here, the mass of ${ }^{133} \mathrm{Cs}$ from the most recent version of the Atomic Mass Evaluation (AME), AME2012 [21], was used. With the frequency ratio $r$, the mass of the atom of interest can be recalculated in the case of an update of the mass of the reference ion. The calculation of statistical and systematic uncertainties has been performed as described in Refs. [22-24]. Most of the resulting ISOLTRAP frequency ratios have already been included in AME2012 [21], which is why we compare our results to those of AME2003 [25]. Conventionally, not masses themselves but mass-excess (ME) values are compared, the latter being calculated via

$$
\operatorname{ME}(Z, N)=m-A \cdot \mathrm{u},
$$

where $A$ denotes the mass number and u the atomic mass unit derived from ${ }^{12} \mathrm{C}$. Therefore, in Table II the individual ISOLTRAP ME values are presented and compared to those from AME2003.

In some cases (see below), a clear distinction of the ground or excited state was not possible or their relative abundance in the beam was not known. In these cases an equal mixture of the two possible states was assumed. Using the energy difference $E$ between the two states, known from elsewhere, the measured $\mathrm{ME}, \mathrm{ME}_{\text {exp }}$, is corrected to yield the estimated ground-state $\mathrm{ME}, \mathrm{ME}_{\mathrm{gs}}$, via

$$
\mathrm{ME}_{\mathrm{gs}}=\mathrm{ME}_{\exp }-\frac{1}{2} E \text {. }
$$

In the calculation of the corresponding uncertainty of the ground-state $\mathrm{ME}, \sigma_{\mathrm{gs}}$, we have to take into account that we just assume an equal mixture. It is hence calculated using the uncertainty of the experimental value $\sigma_{\exp }$ and the uncertainty 
TABLE II. Masses measured with the ISOLTRAP mass spectrometer. The ratios $r$ of the cyclotron frequencies of the reference ion ${ }^{133} \mathrm{Cs}^{+}$ to the ion of interest are listed and the experimental results for mass-excess values are compared to those from AME2003 [25]. \# indicates estimated values; a superscript $x$ unknown isomeric mixtures. In the case of ${ }^{195} \mathrm{Tl}_{114}^{g, m}$ the average ME of the ground state and isomeric state is shown. Masses of ground states labeled with a superscript asterisk $\left(^{*}\right)$ have been corrected according to Ref. [21] to account for unknown mixtures. In the case of ${ }^{198} \mathrm{Tl}$ the isomeric state was measured and the ground state was determined by subtracting the excitation energy given in AME2012. Half-lives are given for information only based on Ref. [27]. Most of the ISOLTRAP results are already included in AME2012. The influence of the data in AME2012 is shown.

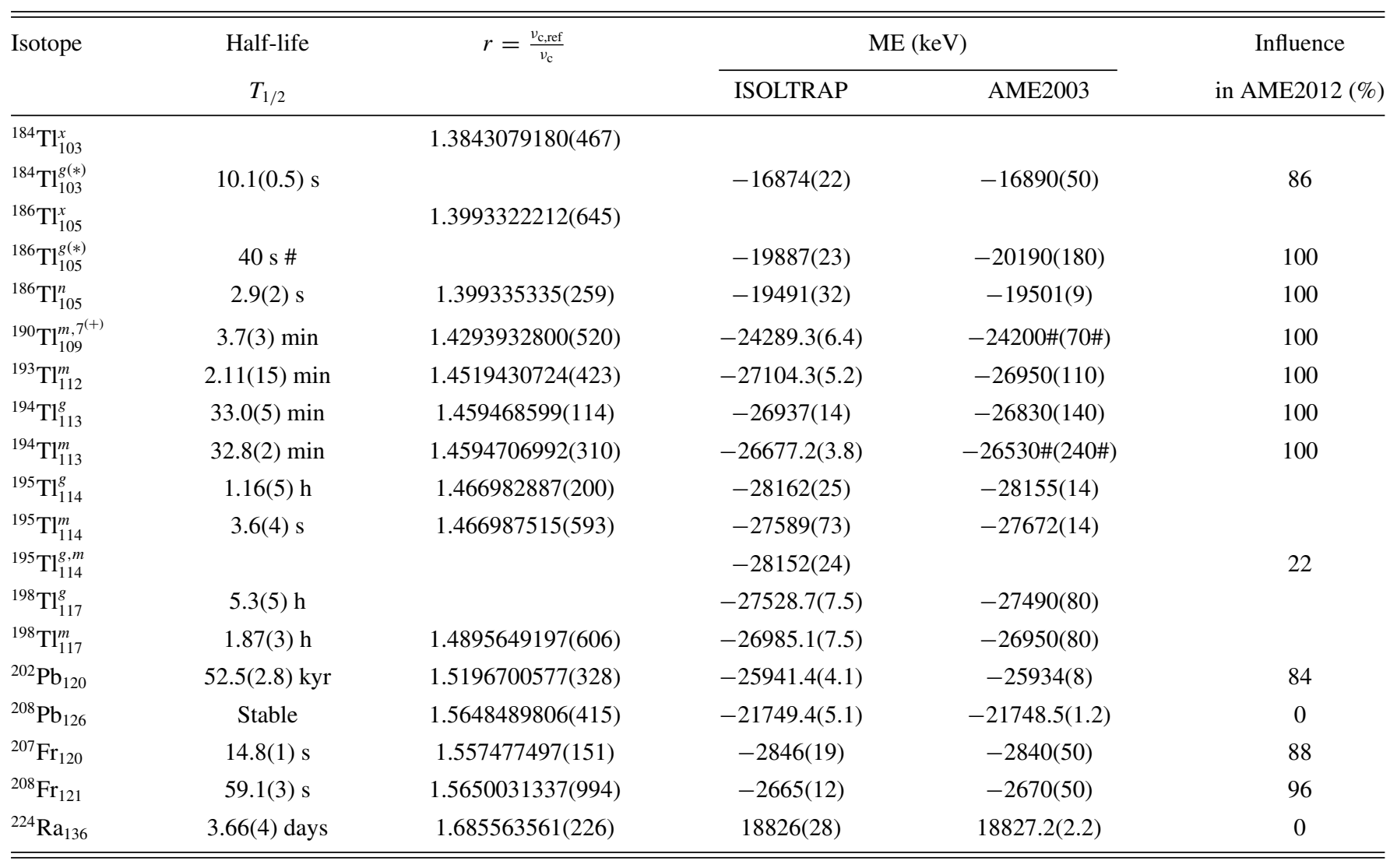

of the energy difference $\sigma_{E}$ via

$$
\sigma_{\mathrm{gs}}=\sqrt{\sigma_{\exp }^{2}+\left(\frac{1}{2} \sigma_{E}\right)^{2}+\frac{1}{12} E^{2}} .
$$

For more details, the reader is referred to Ref. [21]. The results of our measurements and their influence on the mass evaluation can be seen in Table II. Most of the mass values were improved. For ${ }^{194} \mathrm{Tl}$ the ground and the first isomeric states were separately measured for the first time [19].

In total, the masses of seven thallium isotopes have been determined. In the case of ${ }^{184} \mathrm{Tl}$ there is no information available on the possible isomeric states present in the initial beam from ISOLDE. Six TOF resonances taken with excitation times of up to $4 \mathrm{~s}$ show no sign of an isomer. However, a preliminary analysis of experimental data obtained by a different experiment at ISOLDE showed a significant contribution of the ground state as well as the first isomeric state [26]. Hence an equal mixture of both states was assumed in the data analysis.

For ${ }^{186} \mathrm{Tl}$ seven TOF-ICR spectra were recorded with excitation times of between 1.2 and $3 \mathrm{~s}$. Two resonances could be resolved in the spectra. Based on the determined energy difference between the two resonances, one of them was assigned to an unknown mixture of ground state and first isomeric state and the second one was assigned to the second isomeric state.

The mass measurement of ${ }^{190} \mathrm{Tl}$ was carried out in two experimental campaigns ( $\mathrm{C}$ and $\mathrm{F}$ ). In total 16 TOF-ICR spectra with excitation times of up to $5 \mathrm{~s}$ were recorded. The measurements of the experimental campaign $\mathrm{F}$ were combined with a decay-spectroscopy measurement [19]. This allowed unambiguous assignment of the measured mass to the isomeric state $7^{(+)}$, which in turn was determined as the first isomeric state in the latest AME. Using a subsequent ISOLTRAP mass measurement of ${ }^{198} \mathrm{At}(Z=85)$, which is linked to ${ }^{190} \mathrm{Tl}$ by an $\alpha$-decay chain, the mass of the $2^{(-)}$state in ${ }^{190} \mathrm{Tl}$ was determined, establishing the ordering and difference in energy between the two states [19].

For ${ }^{193} \mathrm{Tl}^{m}$ six TOF-ICR spectra, with excitation times of up to $2.5 \mathrm{~s}$, have been recorded. Here, the state assignment was possible due to a decay-spectroscopy measurement [28]. The mass of the first isomeric state of ${ }^{193} \mathrm{Tl}$ was measured for the first time.

In the case of ${ }^{194} \mathrm{Tl}$ six TOF-ICR spectra, with excitation times of up to $4 \mathrm{~s}$, have been recorded and the isomer as well 


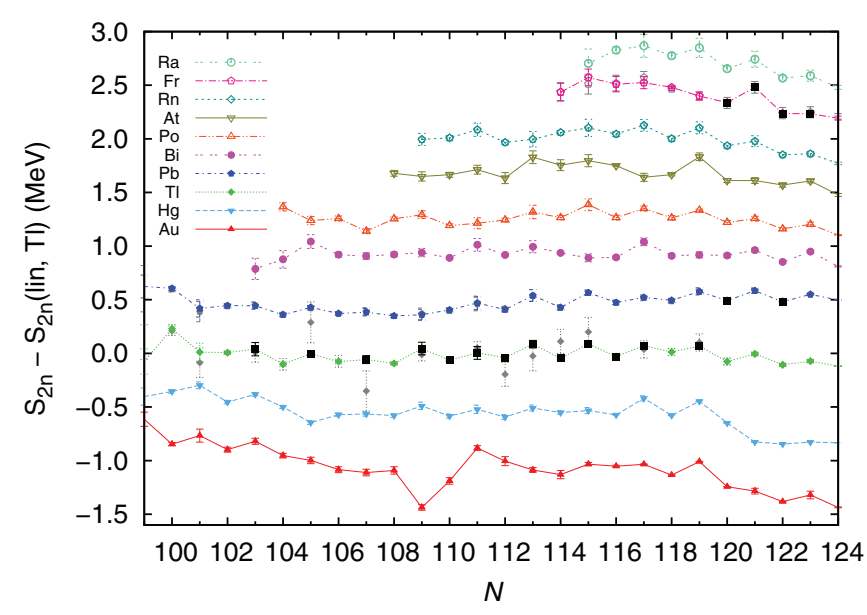

FIG. 2. (Color online) Two-neutron separation energies $S_{2 \mathrm{n}}$ for isotopes in the thallium region as a function of the neutron number $N$. A linear trend, obtained by a linear fit to the $\mathrm{Tl}$ data, was subtracted for better visibility. Data were taken from AME2003 (gray symbols) and AME2012 (colored symbols and lines). New ISOLTRAP results are represented by black squares. The irregularities at ${ }^{186,188} \mathrm{Tl}(N=$ $105,107)$ were already corrected before the present measurements by a re-evaluation of existing data. Initially, mass measurements of the ground state and isomeric state had not been assigned properly. Taking into account the present ISOLTRAP work, a small staggering becomes visible along the $S_{2 \mathrm{n}}$ curve of the Tl isotopic chain.

as the ground state could be separately measured (see Fig. 2 in [19]) for the first time. There are two production channels for this nuclide: a direct one by proton bombardment on a target and an indirect one by decay of heavier species produced in the target. Mainly the first excited state is produced directly, while the ground state is mainly from indirect production. Hence the production ratio of the ground and isomeric states could be influenced by stopping the proton impact on the target. This made the determination of the ground-state mass possible, which is most likely produced by $\beta$ decay from ${ }^{194} \mathrm{~Pb}$. For more details on the spectroscopy measurements performed in experimental campaign D, see Ref. [19].

In the measurement of ${ }^{195} \mathrm{Tl}$, the ground and the isomeric state could be seen. However, as the ground state was much more abundant, additional checks were performed to validate the presence of an isomeric state: Due to the strongly differing half-lives of the isomeric and ground state, one TOF-ICR spectrum was recorded with a $100-\mathrm{ms}$ and another with 1.2-s excitation time, respectively. In these spectra, a significant increase in the number of decay products with increasing excitation time (storage duration) was observed, i.e., ions which arrived at the MCP detector very rapidly due to their higher kinetic energy after decay [29]. This is expected in the case of an isomeric admixture of the 3.6-s isomer. In AME2012, an average of the ground-state and the isomeric value corrected for the known excitation energy is used.

For ${ }^{198} \mathrm{Tl}$ three TOF-ICR spectra of the first isomeric state have been recorded. The excitation time was set to $1.2 \mathrm{~s}$ and the uncertainty could be reduced by approximately a factor of $\sim 10$. Since the measurement was performed in October 2012 the ME of ${ }^{198} \mathrm{Tl}$ is not included in AME2012.
The mass of ${ }^{202} \mathrm{~Pb}$ was determined from 10 TOF-ICR spectra with excitation times of up to $10 \mathrm{~s}$ and the uncertainty could be reduced by a factor of $\sim 2$. For ${ }^{208} \mathrm{~Pb}$ four resonances were taken with an excitation time of $1.2 \mathrm{~s}$. This result has been included in the AME2012 as well, but it does not contribute due to its large uncertainty.

In the case of ${ }^{207} \mathrm{Fr}$ and ${ }^{208} \mathrm{Fr}$, one and five TOF-ICR spectra with 1.2-s excitation times were taken, respectively, and the results are included in AME2012. Furthermore, one spectrum of ${ }^{224} \mathrm{Ra}$ was taken with an excitation time of $1.2 \mathrm{~s}$. Due to its large uncertainty, there is no contribution to AME2012. However, it marks the first direct mass measurement of ${ }^{224} \mathrm{Ra}$, agreeing with the literature value within its uncertainty.

\section{DISCUSSION}

The thallium isotopes have one proton less than the protonmagic lead configuration but, at the same time, have an excess of only two protons compared to the gold isotopes, where deformation sets in towards the neutron midshell from $N=$ 126 to $N=82$. In the following, the systematics of finitedifference mass formulas and differential mean-square nuclear charge radii along the thallium isotopic chain are analyzed, to position them with respect to the spherical and statically deformed limits. Furthermore, the staggering in the two-proton separation energy is addressed.

From the ME value the binding energy of the nucleus $B(Z, N)$ can be calculated as

$$
\begin{aligned}
B(Z, N)= & Z \cdot m_{\mathrm{p}}+N \cdot m_{\mathrm{n}}-[(Z+N) \cdot \mathrm{u} \\
& \left.+\operatorname{ME}(Z, N)-Z \cdot m_{\mathrm{e}}\right],
\end{aligned}
$$

with $m_{\mathrm{p}}, m_{\mathrm{n}}$, and $m_{\mathrm{e}}$ denoting the proton mass, the neutron mass, and the electron mass, respectively. From the binding energy, the two-neutron separation energy can be calculated via

$$
S_{2 \mathrm{n}}(N)=B(Z, N)-B(Z, N-2) .
$$

Nuclear shell effects become apparent as steep drops in $S_{2 n}$ at the crossing of magic numbers. Figure 2 shows a comparison of the $S_{2 n}$ of isotopes in the thallium region as derived from the AME2012 and AME2003 values. The general linear trend was fitted to the $\mathrm{Tl}$ chain and subtracted from the data to reveal subtle effects like the staggering in the case of $\mathrm{Tl}$ and the change in the slope in the Au chain around $N=109$. The $S_{2 \mathrm{n}}$ values from AME2003 exhibited considerable irregularities as noted in a prior analysis of this mass region [30]. With our data, the $S_{2 n}$ curve of the thallium isotopes is smoother and similar in behavior to that of the spherical ground states of lead.

One of the higher-order finite-difference mass formulas is the empirical pairing gap, which is normally used to isolate the strength of the odd-even mass staggering. As a general trend for near-spherical nuclides, the empirical pairing gap exhibits a parabolic shape between magic numbers with a peak at midshell (see, for example, Fig. 3 in Ref. [31]). The three-point empirical pairing gap is given by

$$
\begin{aligned}
\Delta_{\mathrm{n}}^{3}(Z, N)= & \frac{(-1)^{N}}{2}(B(Z, N-1)+B(Z, N+1) \\
& -2 B(Z, N)) .
\end{aligned}
$$




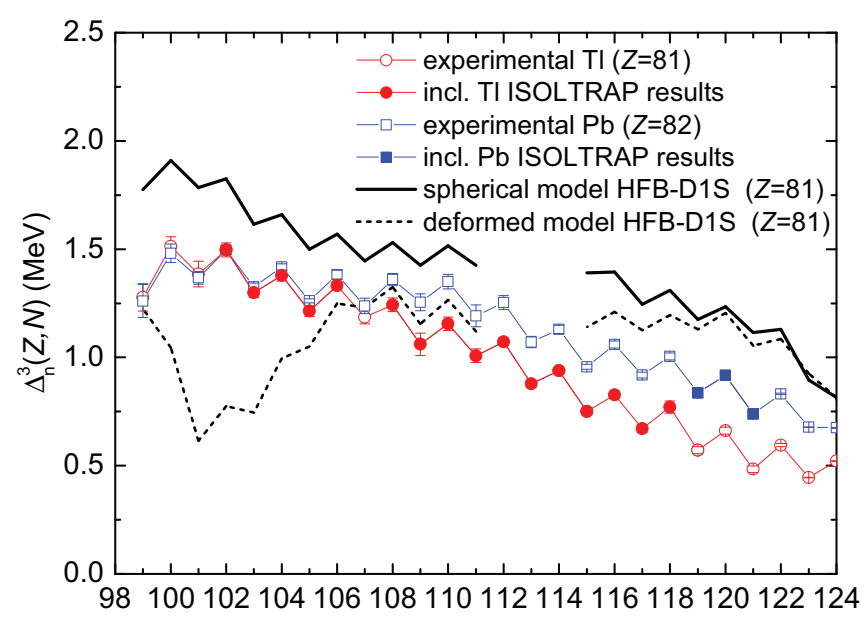

N

FIG. 3. (Color online) Three-point empirical pairing gap $\Delta_{\mathrm{n}}^{3}(Z, N)$ for thallium isotopes as a function of the neutron number $N$. Experimental values [(red) circles] are compared to spherical (solid black line) and deformed (dashed black line) HFB-D1S calculations by the Bruyères-le-Châtel group [37]. Furthermore, $\Delta_{\mathrm{n}}^{3}(Z, N)$ for lead isotopes is shown [(blue) squares]. The binding energy in the HFB-D1S calculation around $N=114$ is not considered since the value likely contains a numerical artifact.

Higher-order finite-difference mass formulas become sensitive to the correlation energy resulting from ground-state collectivity. Because the ground-state correlation energy also affects the odd-even staggering, the pairing gap is sensitive to deformation effects. There have been numerous efforts in mean-field theory to describe the different contributions to the pairing gap [31-36].

The evolution of $\Delta_{\mathrm{n}}^{3}(Z, N)$ from neutron number $N=126$ towards midshell and beyond qualitatively agrees for the $\mathrm{Tl}$ and $\mathrm{Pb}$ isotopic chains as shown in Fig. 3 [(red) circles and (blue) squares, respectively]. In addition to the staggering of $\Delta_{\mathrm{n}}^{3}(Z, N)$, both curves are compatible with the general parabolic shape [31]. In both cases the parabolic shapes peak for smaller $N$ than expected, between $N=102$ and $N=100$. Furthermore, the $\operatorname{dip}$ at $N \approx 105$ in $\Delta_{\mathrm{n}}^{3}(Z, N)$ that appeared in results based on data from 2008 [30] has disappeared. Comparing microscopic calculations using the Hartree-Fock-Bogoliubov (HFB) model in the spherical approximation [37] (solid black line) with $\mathrm{Tl}$ data, we find good agreement. Calculations using the HFB model [37] and including static deformation (dashed black line) clearly show discrepancies to the data around midshell. Like the chain of proton-magic spherical lead isotopes, the empirical pairing gap of the $\mathrm{Tl}$ isotopes shows no evidence of static deformation.

In the following, these first conclusions from experimental masses are compared to differences in mean-square nuclear charge radii. Figure 4 in Ref. [5] shows the difference in mean-square nuclear charge radii $\delta\left\langle r^{2}\right\rangle$ of $\mathrm{Tl}$ isotopes with respect to ${ }^{205} \mathrm{Tl}$, as a function of the mass number $A$. As in the mass values, a slight odd-even staggering is visible from the differences in mean-square nuclear charge radii, as well as a slope change around $N=114$. From a comparison to
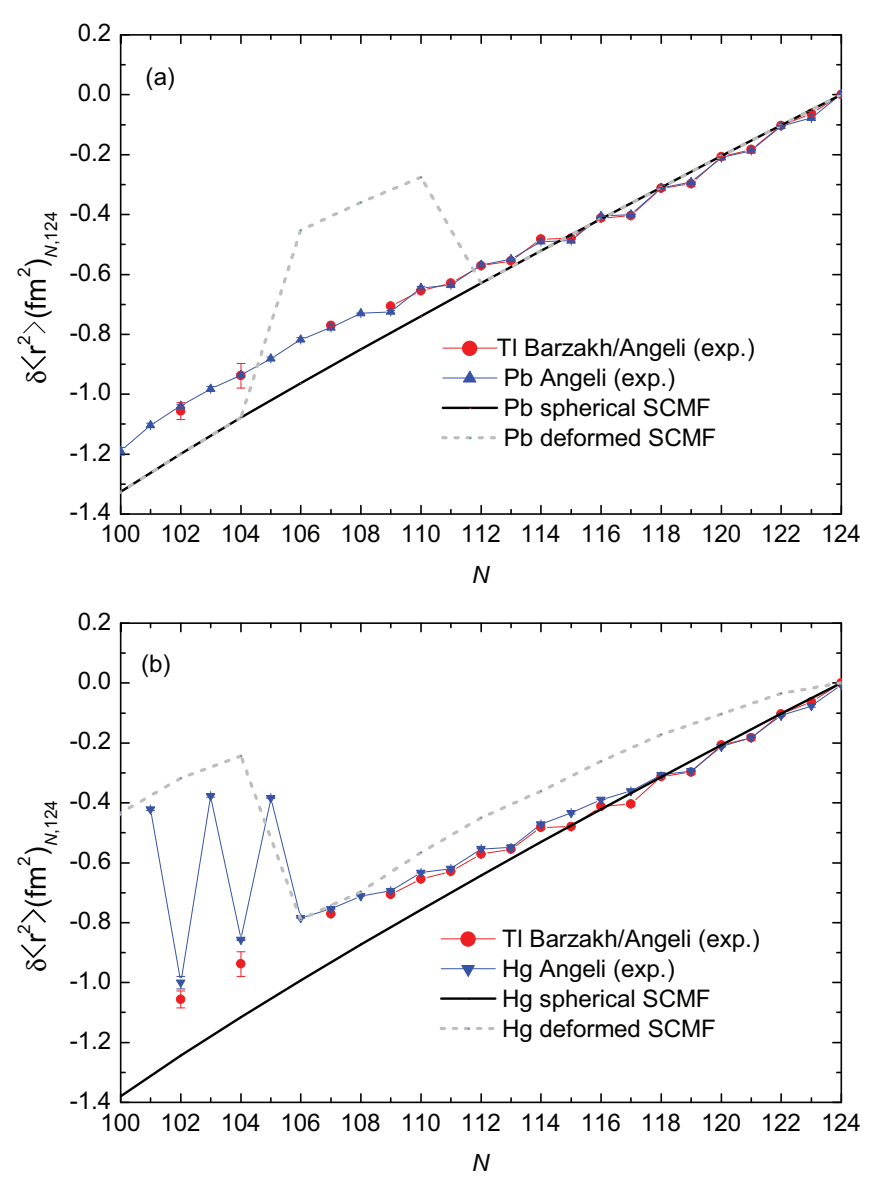

FIG. 4. (Color online) Theoretical values of $\delta\left\langle r^{2}\right\rangle$ [39] are compared to experimental data $[5,40]$. (a) $\mathrm{Tl}$ [(red) circles] compared to $\mathrm{Pb}$ [upward (blue) triangles]; (b) Tl [(red) circles] in comparison to $\mathrm{Hg}$ [downward (blue) triangles]. The black line (spherical case) and the dashed gray line (statically deformed case) denote theoretical results.

the liquid-droplet model [38] the strength of the mean-square quadrupole deformation parameter $\left\langle\beta^{2}\right\rangle^{1 / 2}$ was extracted. Beyond midshell and for $N<113$, the differential meansquare nuclear charge radii lie between the $\left\langle\beta^{2}\right\rangle^{1 / 2}=0$ line and the $\left\langle\beta^{2}\right\rangle^{1 / 2}=0.2$ line.

Next, in Fig. 4 we show the differences in mean-square nuclear charge radii of $\mathrm{Pb}(Z=82)$ [Fig. 4(a)] and $\mathrm{Hg}(Z=$ 80) [Fig. 4(b)], which are compared to those of thallium [(red) circles] and calculations by Bender et al. [39]. Experimental values were taken from [5] and [40]. The differences in mean-square nuclear charge radii are calculated with respect to the reference value at $N=124$. Within a nonrelativistic self-consistent mean-field model, the authors use the Skyrme interaction SLy4 [41] and a density-dependent pairing force to study quadrupole correlation effects. This calculation provides binding energies and mean-square nuclear charge radii for even-even nuclides. The spherical solution (black line) and the statically deformed solution (dashed gray line) of [39] are compared to the data in Fig. 4. As expected, the lead isotopes [Fig. 4(a)] are best described by the spherical solution along the whole isotopic chain. In the case of the $\mathrm{Hg}$ isotopes, 
the spherical solution describes best the data down to about $N=110$. At that point, the deformed solution starts to better describe the data, especially after the onset of strong staggering below $N=106$. Comparing the behavior of the differential mean-square nuclear charge radii of the thallium ground state to that of lead and mercury suggests spherical ground states. Recently the nuclear charge radii of $\mathrm{Tl}$ at $N=103$ and $N=105$ for $I=2$ and $I=7$ were measured [5,6] and follow the trend in Fig. 4, however, the spin-state assignment is still uncertain for both isotopes.

We further investigate whether, contrary to the $\mathrm{Tl}$ isotopes, signs of static deformation can be made visible in higherorder finite-difference mass formulas. The five-point empirical pairing gap given by

$$
\begin{aligned}
\Delta_{\mathrm{n}}^{5}(Z, N)= & \frac{(-1)^{N}}{8}(B(Z, N+2)-4 B(Z, N+1)+6 B(Z, N) \\
& -4 B(Z, N-1)+B(Z, N-2))
\end{aligned}
$$

is shown in Fig. 5 for $\mathrm{Au}$ (black circles), a chain where it is known from the differential mean-square nuclear charge radii that strong deformation sets in at $N \leqslant 107$ [Fig. 5; (red) triangles]. A dip appears in the trend of $\Delta_{n}^{5}(Z, N)$ coinciding with the transition in the differential mean-square nuclear charge radii. However, as shown by Bender et al. through self-consistent mean-field calculations of semimagic nuclides, treating odd-mass nuclides in the spherical blocking approximation [33], the staggering present in the three-point empirical pairing gap should disappear for the symmetric fivepoint empirical pairing gap. If $\Delta_{n}^{5}(Z, N)$ were a pure measure of the pairing gap, this quantity should not respond to a sudden deformation. To show how a sudden onset of deformation can become visible in the empirical pairing gap of Eq. (9), we introduce an expression for describing the different contributions to the binding energy $B(Z, N)$ of a nucleus, which in general

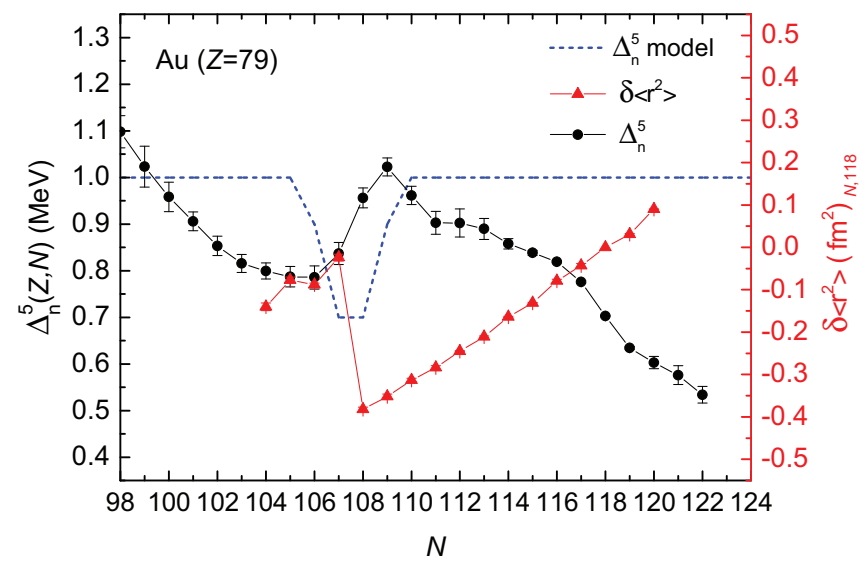

FIG. 5. (Color online) Empirical pairing gap $\Delta_{\mathrm{n}}^{5}(Z, N)$ (black circles) and differential mean-square nuclear charge radii $\delta\left\langle r^{2}\right\rangle$ [(red) triangles] for $\mathrm{Au}$ isotopes as a function of the neutron number $N$. Data were taken from [21] and [40], respectively. Both $\Delta_{\mathrm{n}}^{5}(Z, N)$ and $\delta\left\langle r^{2}\right\rangle$ show irregularities around midshell $(N=104)$. The response of the $\Delta_{\mathrm{n}}^{5}(Z, N)$ model [Eq. (10)] to additional energy of deformation is given by the dashed (blue) line. For details see text. can be decomposed as

$$
\begin{aligned}
B(Z, N)= & B_{\mathrm{sph}}(Z, N)+B_{\mathrm{def}}(Z, N) \\
& -\frac{1+(-1)^{N+1}}{2} \cdot \Delta_{\mathrm{n}}(Z, N) \\
& -\frac{1+(-1)^{Z+1}}{2} \cdot \Delta_{\mathrm{p}}(Z, N) .
\end{aligned}
$$

The total binding energy $B(Z, N)$ is calculated as the sum of a term in the absence of deformation $B_{\mathrm{sph}}(Z, N)$, the contribution from deformation $B_{\text {def }}(Z, N)$, and the pairing gaps of neutrons $\Delta_{\mathrm{n}}(Z, N)$ and protons $\Delta_{\mathrm{p}}(Z, N)$ [42]. For simplicity, Fig. 5 [dashed (blue) line] shows the result of such a decomposition assuming that $B_{\mathrm{sph}}(Z, N)$ is a smooth energy term which does not contribute to $\Delta_{\mathrm{n}}^{5}(Z, N)$ and that the pairing gap $\Delta_{\mathrm{n}}(Z, N)=1 \mathrm{MeV} \forall N$. To simulate a sudden onset of deformation $B_{\text {def }}=0.8 \mathrm{MeV} \forall N \leqslant 107$. Comparing the result [dashed (blue) line] to experimental values (black circles), the dip around $N=107$ can be reproduced by the expression given in Eq. (10). This is in agreement with a jump to larger values in the differential mean-square nuclear charge radii. The same observations can be made for $\Delta_{\mathrm{n}}^{5}(Z, N)$ and the differential mean-square nuclear charge radii along the $\mathrm{Hg}$ isotopic chain (compare Fig. 18 in [30]). We conclude that static deformation can indeed be made visible in the five-point empirical pairing gap. Still, we note that the kink produced by the expression in Eq. (10) is much narrower than the full effect in $\Delta_{\mathrm{n}}^{5}(Z, N)$. In contrast, the smooth behavior of $\Delta_{\mathrm{n}}^{3}(Z, N)$ (Fig. 3) and, accordingly, $\Delta_{\mathrm{n}}^{5}(Z, N)$ (Fig. 6) of the Tl isotopes signals the absence of static deformation for $N \geqslant 100$.

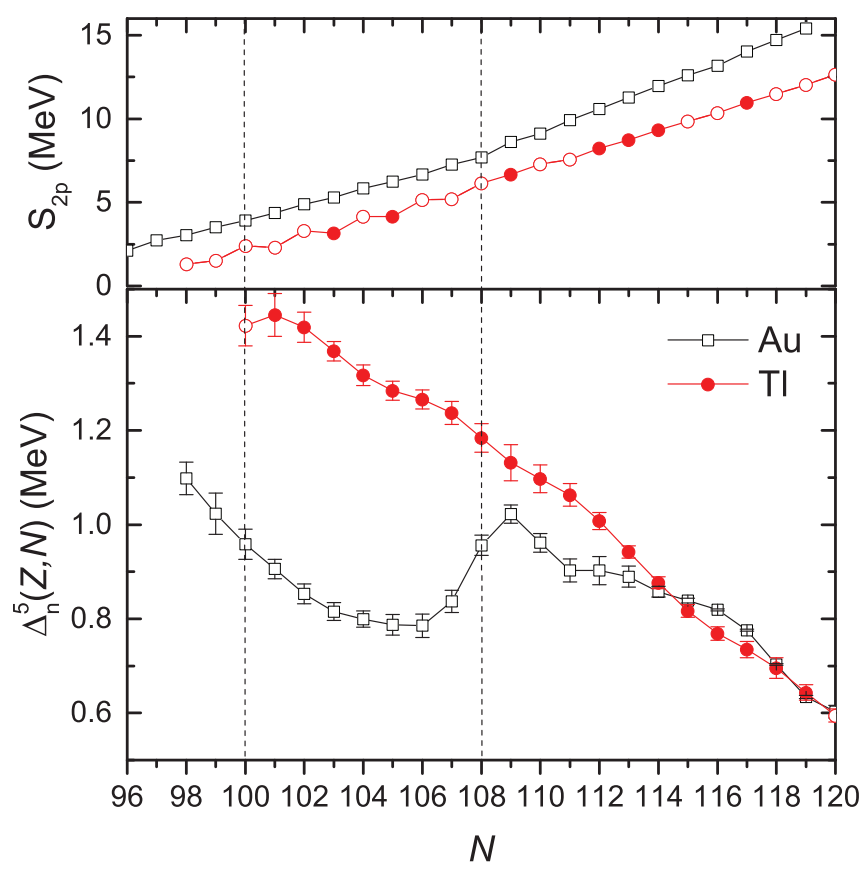

FIG. 6. (Color online) Two-proton separation energies (top) and five-point empirical pairing gaps (bottom) of $\mathrm{Tl}$ [(red) circles] and $\mathrm{Au}$ [(black) squares] isotopes. Data are taken from AME2012 and include the new ISOLTRAP results (filled circles). For two-proton separation energies, error bars are smaller than the symbol size. 
While the effect of the removal of neutrons has been highlighted with the $S_{2 n}$ and associated mass formulas, the effect of the valence proton in $\mathrm{Tl}$ isotopes can be discussed in terms of the two-proton separation energy. In analogy to $S_{2 n}$, one looks at the two-proton-separation energy given by

$$
S_{2 \mathrm{p}}(Z, N)=B(Z, N)-B(Z-2, N) .
$$

Figure 6 shows in parallel the two proton separation energies and the five-point empirical pairing gaps in the $\mathrm{Tl}$ and $\mathrm{Au}$ chain. The $S_{2 \mathrm{p}}$ values of Tl exhibit a strong staggering from $N=$ 100 to $N=108$, which is unexpected for this observable. To investigate the possible origin of this uncommon phenomenon, we rephrase $S_{2 p}$ by using Eq. (10):

$$
\begin{aligned}
S_{2 \mathrm{p}}(Z, N)= & S_{2 \mathrm{p}, \mathrm{sph}}(Z, N)+S_{2 \mathrm{p}, \mathrm{def}}(Z, N) \\
& +\frac{1+(-1)^{N+1}}{2} \cdot\left[\Delta_{\mathrm{n}}(Z-2, N)-\Delta_{\mathrm{n}}(Z, N)\right] \\
& +\frac{1+(-1)^{Z+1}}{2} \cdot\left[\Delta_{\mathrm{p}}(Z-2, N)-\Delta_{\mathrm{p}}(Z, N)\right],
\end{aligned}
$$

which shows that $S_{2 p}$ shows a staggering with respect to $N$ and with respect to $Z$, equal to the variation between $Z$ and $Z-2$ of the neutron and proton pairing gaps, respectively. The strong variation of the neutron pairing gap $\Delta_{\mathrm{n}}(Z, N)$ between the Tl $(Z=81)$ and the $\mathrm{Au}(Z=79)$ isotopic chains in the region $100 \leqslant N \leqslant 108$ indeed produces a significant staggering of $S_{2 \mathrm{p}}$ with respect to $N$, in the same region.

Further information about the development of collectivity along the $\mathrm{Tl}$ isotopic chain can be extracted from the spectroscopy of low-lying isomeric states (Fig. 7). In general, for $103 \leqslant N \leqslant 121$ the odd- $N$ Tl isotopes exhibit a $\left(2^{-}\right)$ ground state and a $\left(7^{+}\right)$isomeric state, while in the even- $N$ $\mathrm{Tl}$ isotopes a $\left(9 / 2^{-}\right)$isomer can be found. The experimental observed level spacing between the $\left(2^{-}\right)$and the $\left(7^{+}\right)$state is discussed in [19] and led to the conclusion of an oversimplified description of the spherical shell model of the $\mathrm{Tl}$ isotopes. Slight oblate deformation or shape coexistence could explain the state ordering [19]. For the even- $N$ Tl isotopes the spherical shell model predicts that the proton occupies the $s_{1 / 2}$ orbital.

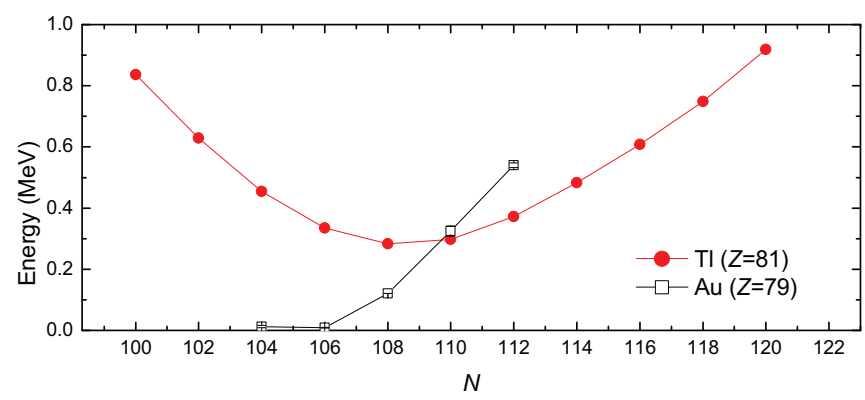

FIG. 7. (Color online) Excitation energy of the $\left(9 / 2^{-}\right)$isomer of even- $N \mathrm{Tl}$ isotopes [filled (red) circles] compared to the excitation energy of the $\left(9 / 2^{-}\right)$state in even- $N$ Au isotopes [open (black) squares]. Data were taken from [21] and [43]. The energy of the $\mathrm{Tl}$ isomers reaches a minimum for $N=108$. The $\left(9 / 2^{-}\right)$isomer reflects the evolution of the $h_{9 / 2}$ intruder, which is responsible for deformation in the gold chain. In the case of Tl, error bars are smaller than the symbol size.
However, numerous $\left(9 / 2^{-}\right)$isomers have been identified, suggesting the proton occupying the $h_{9 / 2}$ orbital.

The $\left(9 / 2^{-}\right)$isomeric state, exhibiting deformation for $N \leqslant$ 116 [5], comes closer to the ground state with decreasing $N$, with a minimal excitation energy of less than $300 \mathrm{keV}$ at $N=$ 108 [see Fig. 7; filled (red) circles]. The $\left(9 / 2^{-}\right)$isomer reflects the evolution of the $h_{9 / 2}$ intruder from above the $Z=82$ shell, the lowering of which is responsible for deformation in the gold chain. In this chain the excitation energy of the $\left(9 / 2^{-}\right)$ state (open black squares) is almost 0 at $N=106$. The slight deviation from spherical behavior of the $\mathrm{Tl}$ ground state could thus be understood as the effect of ground-state correlations, also reflected in the presence of the low-lying intruder state.

\section{CONCLUSION}

Masses of neutron-deficient thallium isotopes have been measured with the Penning-trap mass spectrometer ISOLTRAP. The structure of this isotopic chain lying between the proton-magic lead isotopes and the well-deformed gold and mercury isotopes has been studied. Looking at the groundstate properties of $\mathrm{Tl}$ using the two-neutron separation energy and the differences in mean-square nuclear charge radii, we find a smooth behavior compatible with similar observations for the lead isotopes. Microscopic calculations assuming a spherical shape of the ground state also agree with the data. The increased precision of nuclear masses allows the investigation of subtle effects such as the influence of deformation on the empirical pairing gap and the impact of the evolution of the pairing gap on the staggering of $S_{2 p}$. As such, it was possible to correlate the sudden onset of deformation in the gold isotopes to a dip in $\Delta_{\mathrm{n}}^{5}(Z, N)$, usually thought of as describing the pairing gap. We have also shown and justified that in cases in which the pairing gap shows a sharp variation with proton number, the two-proton separation energy exhibits an enhanced staggering, which is a direct reflection of this variation. We conclude that in the $\mathrm{Tl}$ isotopes, the role of the intruder $h_{9 / 2}$ orbital is not strongly pronounced. The systematics of the excitation energy of the $I=(9 / 2)(\mathrm{Tl})$ isomer supports this picture, since the $\left(9 / 2^{-}\right)$level never becomes the ground state, but the parabolic shape of the excitation energy shows that collectivity is already present in the thallium isotopic chain.

\section{ACKNOWLEDGMENTS}

The authors thank the ISOLDE Collaboration and the ISOLDE Technical Group for their support. We thank Anatoly Barzakh for discussions about nuclear charge radii of $\mathrm{Tl}$ isotopes. We also thank Jacek Dobaczewski for discussion of empirical pairing gaps. This work was supported by the German Federal Ministry for Education and Research (BMBF) (Grant Nos. 05P09ODCIA, 05P09HGFNE, 05P12HGCI1, 05P12HGFNE, and 06MZ215), a Marie Curie Actions grant (No. MEIF-CT-2006-042114, EU FP6), the Max-Planck Society, the EU FP7 through ENSAR (Contract No. 262010), and the French IN2P3. R.B.C. acknowledges support by the MaxPlanck-Partner group. T.E.C. was supported by the UK Science and Technology Facilities Council. S.K. acknowledges support from the Robert-Bosch Foundation within the Fast Track Program. 
[1] V. Manea, D. Atanasov, D. Beck, K. Blaum, Ch. Borgmann, R. B. Cakirli, T. Eronen, S. George, F. Herfurth, A. Herlert, M. Kowalska, S. Kreim, Y. A. Litvinov, D. Lunney, D. Neidherr, M. Rosenbusch, L. Schweikhard, F. Wienholtz, R. N. Wolf, and K. Zuber, Phys. Rev. C 88, 054322 (2013).

[2] K. Heyde and J. L. Wood, Rev. Mod. Phys. 83, 1467 (2011).

[3] J. Bonn, G. Huber, H.-J. Kluge, L. Kugler, and E. Otten, Phys. Lett. B 38, 308 (1972).

[4] A. Andreyev, M. Huyse, P. van Duppen, L. Weissman, D. Ackermann, J. Gerl, F. P. Heßberger, S. Hofmann, A. Kleinböhl, G. Münzenberg, S. Reshitko, C. Schlegel, H. Schaffner, P. Cagarda, M. Matos, S. Saro, A. Keenan, C. Moore, C. D. O'Leary, R. D. Page, M. Taylor, H. Kettunen, M. Leino, A. Lavrentiev, R. Wyss, and K. Heyde, Nature 405, 430 (2000).

[5] A. E. Barzakh, L. K. Batist, D. V. Fedorov, V. S. Ivanov, K. A Mezilev, P. L. Molkanov, F. V. Moroz, S. Y. Orlov, V. N. Panteleev, and Y. M. Volkov, Phys. Rev. C 88, 024315 (2013).

[6] H. A. Schuessler, E. C. Benck, F. Buchinger, and H. K. Carter, Nucl. Instrum. Methods Phys. Res. A 352, 583 (1995).

[7] D. G. Madland and J. R. Nix, Nucl. Phys. A 476, 1 (1988).

[8] M. Mukherjee, D. Beck, K. Blaum, G. Bollen, J. Dilling, S. George, F. Herfurth, A. Herlert, A. Kellerbauer, H.-J. Kluge, S. Schwarz, L. Schweikhard, and C. Yazidjian, Eur. Phys. J. A 35, 1 (2008).

[9] S. Kreim, D. Atanasov, D. Beck, K. Blaum, Ch. Böhm, Ch. Borgmann, M. Breitenfeldt, T. E. Cocolios, D. Fink, S. George, A. Herlert, A. Kellerbauer, U. Köster, M. Kowalska, D. Lunney, V. Manea, E. Minaya Ramirez, S. Naimi, D. Neidherr, T. Nicol, R. E. Roessel, M. Rosenbusch, L. Schweikhard, J. Stanja, F. Wienholtz, R. N. Wolf, and K. Zuber, Nucl. Instrum. Methods Phys. Res. B 317, 492 (2013).

[10] E. Kugler, Hyp. Int. 129, 23 (2000).

[11] H.-Jürgen Kluge, Int. J. Mass Spectrom. 349-350, 26 (2013).

[12] K. Blaum, J. Dilling, and W. Nörtershäuser, Physica Scripta 2013, 014017 (2013).

[13] F. Herfurth, J. Dilling, A. Kellerbauer, G. Bollen, S. Henry, H.-J. Kluge, E. Lamour, D. Lunney, R. B. Moore, C. Scheidenberger, S. Schwarz, G. Sikler, and J. Szerypo, Nucl. Instrum. Methods Phys. Res. A 469, 254 (2001).

[14] R. N. Wolf, F. Wienholtz, D. Atanasov, D. Beck, K. Blaum, Ch. Borgmann, F. Herfurth, M. Kowalska, S. Kreim, Yu. A. Litvinov, D. Lunney, V. Manea, D. Neidherr, M. Rosenbusch, L. Schweikhard, J. Stanja, and K. Zuber, Int. J. Mass Spectrom. 349-350, 123 (2013).

[15] G. Savard, St. Becker, G. Bollen, H. J. Kluge, R. B. Moore, T. Otto, L. Schweikhard, H. Stolzenberg, and U. Wiess, Phys. Lett. A 158, 247 (1991).

[16] G. Gräff, H. Kalinowsky, and J. Traut, Z. Phys. A 297, 35 (1980).

[17] M. König, G. Bollen, H.-J. Kluge, T. Otto, and J. Szerypo, Int. J. Mass. Spectrom. Ion Process. 142, 95 (1995).

[18] M. Kowalska, S. Naimi, J. Agramunt, A. Algora, D. Beck, B. Blank, K. Blaum, Ch. Böhm, Ch. Borgmann, M. Breitenfeldt, L. Fraile, S. George, F. Herfurth, A. Herlert, S. Kreim, D. Lunney, E. Minaya-Ramirez, D. Neidherr, M. Rosenbusch, B. Rubio, L. Schweikhard, J. Stanja, and K. Zuber, Nucl. Instrum. Methods Phys. Res. A 689, 102 (2012).

[19] J. Stanja, Ch. Borgmann, J. Agramunt, A. Algora, D. Beck, K. Blaum, Ch. Böhm, M. Breitenfeldt, T. E. Cocolios, L. M. Fraile,
F. Herfurth, A. Herlert, M. Kowalska, S. Kreim, D. Lunney, V. Manea, E. Minaya Ramirez, S. Naimi, D. Neidherr, M. Rosenbusch, L. Schweikhard, G. Simpson, F. Wienholtz, R. N. Wolf, and K. Zuber, Phys. Rev. C 88, 054304 (2013).

[20] S. Sturm, F. Köhler, J. Zatorski, A. Wagner, Z. Harman, G. Werth, W. Quint, C. H. Keitel, and K. Blaum, Nature 467-470, 506 (2014).

[21] G. Audi, M. Wang, A. H. Wapstra, F. G. Kondev, M. MacCormick, X. Xu, and B. Pfeiffer, Chin. Phys. C 36, 1287 (2012).

[22] A. Kellerbauer, K. Blaum, G. Bollen, F. Herfurth, H.-J. Kluge, M. Kuckein, E. Sauvan, C. Scheidenberger, and L. Schweikhard, Eur. Phys. J. D 22, 53 (2003).

[23] Ch. Borgmann, Ph.D. thesis, Ruprecht Karls-Universität Heidelberg, 2012; http://www.ub.uni-heidelberg.de/archiv/13925

[24] A. T. Gallant, M. Brodeur, T. Brunner, U. Chowdhury, S. Ettenauer, V. V. Simon, E. Mané, M. C. Simon, C. Andreoiu, P. Delheij, G. Gwinner, M. R. Pearson, R. Ringle, and J. Dilling, Phys. Rev. C 85, 044311 (2012).

[25] A. H. Wapstra, G. Audi, and C. Thibault, Nucl. Phys. A 729, 129 (2003).

[26] E. Rapisarda (private communication).

[27] G. Audi, F. G. Kondev, M. Wang, B. Pfeiffer, X. Sun, J. Blachot, and M. MacCormick, Chin. Phys. C 36, 1157 (2012).

[28] J. Stanja, Ph.D. thesis, Technische Universität Dresden, 2012; http://nbn-resolving.de/urn:nbn:de:bsz:14-qucosa-113289

[29] A. Herlert, S. Van Gorp, D. Beck, K. Blaum, M. Breitenfeldt, R. Cakirli, S. George, U. Hager, F. Herfurth, A. Kellerbauer, D. Lunney, R. Savreux, L. Schweikhard, and C. Yazidjian, Eur. Phys. J. A 48, 97 (2012).

[30] C. Weber, G. Audi, D. Beck, K. Blaum, G. Bollen, F. Herfurth, A. Kellerbauer, H.-J. Kluge, D. Lunney, and S. Schwarz, Nucl. Phys. A 803, 1 (2008).

[31] J. Dobaczewski, P. Magierski, W. Nazarewicz, W. Satula, and Z. Szymanski, Phys. Rev. C 63, 024308 (2001).

[32] W. Satula, J. Dobaczewski, and W. Nazarewicz, Phys. Rev. Lett. 81, 3599 (1998).

[33] M. Bender, K. Rutz, P.-G. Reinhard, and J. A. Maruhn, Eur. Phys. J. A 8, 59 (2000).

[34] T. Duguet, P. Bonche, P.-H. Heenen, and J. Meyer, Phys. Rev. C 65, 014310 (2001).

[35] T. Duguet, P. Bonche, P.-H. Heenen, and J. Meyer, Phys. Rev. C 65, 014311 (2001).

[36] L. M. Robledo, R. Bernard, and G. F. Bertsch, Phys. Rev. C 86, 064313 (2012)

[37] S. Hilaire and M. Girod, Eur. Phys. J. A 33, 237 (2007).

[38] D. Berdichevsky and F. Tondeur, Z. Phys. A 322, 141 (1985).

[39] M. Bender, G. F. Bertsch, and P.-H. Heenen, Phys. Rev. C 73, 034322 (2006).

[40] I. Angeli and K. P. Marinova, At. Data Nucl. Data Tables 99, 69 (2013).

[41] E. Chabanat, P. Bonche, P. Haensel, J. Meyer, and R. Schaeffer, Nucl. Phys. A 635, 231 (1998).

[42] We note that $\Delta_{\mathrm{n}}(Z, N)$ and $\Delta_{\mathrm{p}}(Z, N)$ include all effects which influence the odd-even staggering of binding energies. We, however, denominate them pairing gaps because the effect of breaking a pair of nucleons is the main contribution.

[43] ENSDF, http://www.nndc.bnl.gov/ensdf/ (2012). 